\title{
How to Choose "Last Mile” Delivery Modes for E-Fulfillment
}

\author{
Xuping Wang, ${ }^{1,2}$ Linmin Zhan, ${ }^{1}$ Junhu Ruan, ${ }^{1,3}$ and Jun Zhang ${ }^{1}$ \\ ${ }^{1}$ Institute of Systems Engineering, Dalian University of Technology, Dalian 116024, China \\ ${ }^{2}$ School of Business, Dalian University of Technology, Panjin 124221, China \\ ${ }^{3}$ School of Electrical and Electronic Engineering, Faculty of Engineering, Computer and Mathematical Sciences, University of Adelaide, \\ Adelaide, SA 5005, Australia
}

Correspondence should be addressed to Junhu Ruan; junhu.ruan@adelaide.edu.au

Received 11 February 2014; Revised 1 May 2014; Accepted 15 May 2014; Published 11 June 2014

Academic Editor: Kwok-Wo Wong

Copyright (c) 2014 Xuping Wang et al. This is an open access article distributed under the Creative Commons Attribution License, which permits unrestricted use, distribution, and reproduction in any medium, provided the original work is properly cited.

"Last mile" delivery has become one of the bottlenecks of e-logistics. This paper aims to explore the competitiveness of three "Last mile" delivery modes - attended home delivery (AHD), reception box (RB), and collection-and-delivery points (CDPs) in different scenarios, especially in high population density scenario. The advantages and disadvantages of each mode are introduced first. Then each mode's operation efficiency is solved with different kinds of vehicle routing problem (VRP) models and genetic algorithm (GA). Finally the cost of each mode is calculated on the basis of cost structures and operation efficiencies. The results show that different modes are suitable for different scenarios: (i) AHD and independent reception box work better in a scenario with sparse population or small order quantity; (ii) shared reception box and CDPs are more appropriate in the scenario with high population density and large order quantity, and the better one depends on the cost of labors and facilities; (iii) RB is desirable in some circumstances as delivering fresh vegetables and fruits to the ones living in high-grade communities.

\section{Introduction}

E-commerce develops rapidly in recent years [1]. According to the data from China Internet Network Information Center, China has the second biggest online shopping market as its online sales broke through 1.2 trillion in 2012 [2]. Ecommerce can hardly do without the support of e-logistics. The "last mile" delivery which accounts for $30 \%$ of total e-logistics cost [3] has become one of the bottlenecks of e-commerce. Nowadays, attended home delivery (AHD), reception box (RB), and collection-and-delivery points (CDPs) are the three existing "last mile" delivery modes in China. This paper mainly explores the competitiveness of three delivery modes in different scenarios, especially in high-density areas, in view of China's high population density. High population density leads to high residential density $[4,5]$. Population density has an important effect on delivery efficiency [6].

Currently, AHD-couriers send goods to customers' doorsteps, receive customers' signatures, and leave for the next one-is the most widespread "last mile" delivery mode.
AHD provides the opportunities with customers face to face, but its low operation efficiency is likely to make it undesirable to handle massive orders. Most researchers focused on the balance between cost and customer satisfaction. Narrow delivery time slots are more popular with customers, but they greatly reduce routing efficiency and lead to a huge cost [7]. Hill et al. made explicit tradeoff between the benefits of short delivery time guarantees and the cost of late deliveries [8]. The concept of optimal position of the delivery window into a cost-based delivery performance model was introduced in [9]. Further, Campbell and Savelsbergh concentrated on using time slot incentives to cut down delivery cost and improve profits [10]. In AHD, couriers always have to waste a lot of time waiting for customers, so RB and CDPs are there in the environment.

There are three kinds of reception boxes: (1) independent reception box is installed at the garage or home yard of the customer; (2) delivery box is equipped with a docking mechanism and will be retrieved after goods inside are taken away; (3) shared reception box is installed near customers for their shared usage. Couriers put goods in the boxes, and customers 
pick up goods at any time using messaged passwords. In this way, couriers are released from time limitation while their delivery efficiencies are improved. Moreover, RB could be used as a refrigerated cabinet to store organic vegetables and fruits. However, the investment of reception boxes is large. Punakivi et al. compared AHD and RB from the perspective of cost and payback period. They found that up to $60 \%$ cost reductions are achievable by using $\mathrm{RB}$, and the operational cost of delivery box is on the same level as the reception box when delivery boxes are not picked up until the next delivery time [11]. Further, Punakivi and Tanskanen found that cost can be saved as high as $55 \%-60 \%$ by using shared reception box compared to the concept offering attended reception with two-hour time windows. However, this mode is only suited to customers living in blocks of flats [12].

Collection-and-delivery points refer to convenience stores, plot properties, and other institutions which belong to or cooperate with express companies as the place where customers come to pick up goods. Couriers just scan and put goods in certain places in CDPs. Operation efficiency is improved dramatically, because a CDP could serve several delivery points at the same time. When CDPs are located at areas that already generate consumer trips, their satisfaction will seldom or never be affected seriously because little additional travel by consumers will be required to collect a package [13]. Lee and Whang suggested CDPs are one of the five best ways to solve "last mile" delivery problem [14]. Different distribution of the third institutions has important influences on delivery cost and environment [15]. Customer mileage could be reduced by eighty percent by using CDPs for failed first-time home shopping deliveries [16]. Besides, CDPs is beneficial to the cooperating parties, because about $25 \%$ CDPs users would make a purchase when collecting or returning their parcels [13].

Different delivery modes have different characteristics and delivery efficiencies. The choice of the most appropriate "last mile" delivery mode in different scenarios has no quantitative guidance. This paper reveals the variations of three delivery modes' cost and operation efficiencies in different scenarios.

The rest of the paper is organized as follows. In Section 2, the method is proposed to calculate the total cost of different delivery modes. Each mode's operation efficiency is solved with VPR models and genetic algorithm. Then on the basis of operation efficiencies and cost structures analyzed, the total cost of each mode is calculated. In Section 3, the specific experiment is conducted and the competitiveness of each mode is analyzed. At last, the conclusions are drawn and further research directions are raised.

\section{Method}

This section proposes a method to calculate the total cost of different "last mile" delivery modes. First, some assumptions are made to define this problem. Second, VRP models are built to achieve operation efficiencies according to each mode's characteristics and genetic algorithm is adopted to solve the VRP problems. Last, the cost structures are proposed to calculate the total cost of each delivery mode together with operation efficiencies.

2.1. Assumptions. Some assumptions are made according to the status quo of "last mile" operation in China as follows.

(1) Packages will not be sent twice. Couriers will get in touch with the receivers before they start to deliver the customers' goods. Actually, first-delivery failure is rare in China.

(2) Reception box employed in this paper is communal reception box with drawers of different sizes. It uses luggage locker technology and PIN codes to control the delivery. It is assumed all the goods could be loaded into the boxes. In rural and suburban areas, most people live in single detached family. Independent reception box and delivery box are desirable in this case because an important advantage of $\mathrm{RB}$ is that customers could get goods nearby. However, in highdensity area, most families live in blocks of flats; thus, communal reception box is preferable [12].

(3) The collection-and-delivery points employed in this paper are convenience stores, supermarkets, subway stations, and some other institutions which cooperate with express companies. In reality, CDPs could be built in two ways: setting up CDPs by express companies themselves and collaborating with third parties [15]. The former one requires more investment and bears more risk. Thus it is rational for express companies to cooperate with other organizations in the early stage.

(4) CDPs and reception boxes are set up abiding strictly by the principles listed below: (i) CDPs should be located within a five-minute driving distance by car from customer address [15]; (ii) each community (a delivery point stands for a community in this paper) - a group of people who live in the same areahas one reception box at least to guarantee customer satisfaction from the view of fair [17]; (iii) CDPs should be accessible to the major road network and parking should be permitted and easy.

2.2. Delivery Efficiency. Different "last mile" delivery modes have different delivery efficiencies. Measuring the operation efficiency of each mode is fundamental for calculating its total cost. Operation efficiency could be measured by average delivery time per order $t_{j}$ with $j$ distinguishing different modes. The larger the $t_{j}$ is, the lower the operation efficiency is.

According to delivery process, no matter which mode it is, total delivery time per day consists of three parts as formula (1) shows: time spent on the road $t_{j}^{\text {road }}$, time spent starting or stopping the vehicle $t_{j}^{s}$, and time spent serving customers $t_{j}^{\text {service }}$, respectively. $t_{j}^{\text {service }}$ indicates time spent walking to customers' doorstep, waiting for consignees' signatures, scanning bar codes, unpacking and inspecting goods in AHD; time spent walking to the places, putting 
goods into appropriate drawers, and scanning bar codes in RB; time spent unloading and scanning bar codes in CDPs.

Therefore, total time spent per day could be

$$
t_{j}^{\mathrm{sum}}=t_{j}^{\mathrm{road}}+t_{j}^{s}+t_{j}^{\mathrm{service}}
$$

The calculation of $t_{j}^{s}$ and $t_{j}^{\text {service }}$ is simpler than $t_{j}^{\text {road }}$; thus the calculation of $t_{j}^{s}$ and $t_{j}^{\text {service }}$ is introduced first. $t_{j}^{s}$ is the average time of parking multiplied by parking times. $t_{j}^{\text {service }}$ is average service time per order multiplied by the number of orders. The formulae of $t_{j}^{\text {service }}$ in three modes are exactly the same as $t_{j}^{s}$.

The process of calculating the value of $t_{j}^{\text {road }}$ is much more complex. In AHD, it is a typical VRPTW (vehicle routing problem with time window) problem in view of a few customers' requirements of delivery time windows [18-21], in $\mathrm{RB}$, it is a VRP problem [22-24], and in CDPs, it is a VRP problem on the basis of cluster analysis [25-28]. Details and basis of modeling are introduced below.

Here is the VRPTW model of AHD. Notations to be used in the VRPTW model are defined as follows.

$R:$ The set of routes

$m$ : The set of delivery points

$d_{i j}$ : The distance between delivery point $i$ and delivery point $j$

$q_{i}$ : Daily demand of delivery point $i$

$q$ : Total daily demand of this region

$q_{0}, q_{n+1}:$ The demand of depot; $q_{0}=q_{n+1}=0$

$Q_{v}:$ The capacity of a vehicle

$\lambda$ : The time spent on serving a customer

$\sigma$ : The time spent on starting and stopping a vehicle

$v$ : Average velocity

$T$ : The daily working time

$x_{i j q}: \operatorname{Arc}(i, j)$ appears in route $r$

$y_{i r}$ : Delivery point $i$ is served by route $r$

$s_{i}$ : The starting time of serving delivery point $i$

$\left[a_{i}, b_{i}\right]$ : The delivery time window of delivery point $i$.

The formula could be as follows.

Objective function:

$$
\min t^{\mathrm{road}}=\sum_{r=1}^{R} \sum_{i=0}^{m+1} \sum_{j=0}^{m+1} x_{i j r}\left(\frac{d_{i j}}{v}\right),
$$

subject to

$$
\begin{gathered}
\sum_{i=1}^{m} q_{i}=q ; \\
\sum_{r=1}^{R} y_{i r}=1 \quad i=1,2,3, \ldots, m ; \\
\sum_{i=0}^{m} x_{i j r}=y_{j r} \quad j=1,2, \ldots, m+1 ; r=1,2, \ldots, R ; \\
\sum_{j=1}^{m+1} x_{i j r}=y_{i r} \quad i=0,1,2, \ldots, m ; r=1,2, \ldots, R ; \\
\sum_{j=1}^{m+1} x_{0 j r}=1 \quad r=1,2, \ldots, R ; \\
\sum_{i=0}^{m} x_{i(m+1) r}=1 \quad r=1,2, \ldots, R ; \\
\sum_{i=1}^{m} y_{i r} q_{i} \leq Q_{v} \quad r=1,2, \ldots R ; \\
s_{i}+\lambda q_{i}+\sigma+\frac{d_{i j}}{v}-M\left(1-\sum_{r=1}^{R} x_{i j r}\right) \leq s_{j} \\
i=0,1,2, \ldots, m ; \quad j=1,2, \ldots, m+1 ; \\
a_{i} \leq s_{i} \leq b_{i} \leq T \quad i=0,1,2, \ldots, m+1 ; \\
x_{i j r} \in\{0,1\}, \quad \begin{array}{l}
j \\
y_{i r}
\end{array}
\end{gathered}
$$

Constraint (3) states that the sum of delivery points' demand cannot be more than total daily demand in this district. Constraints (4)-(6) state that every delivery point should be visited at most once. Constraints (7)-(8) are flow conservation constraints that describe the individual routes. Constraint (9) states that the total demand on a route cannot be more than vehicle capacity. Constraints (10) and (11) ensure the feasibility of the time schedule.

Real-coded genetic algorithm which is one of the most effective ways to solve VRPTW problems is employed in this paper. Sequence coding is used and each delivery point is assigned a serial number, and 0 represents the depot. A chromosome consists of $m+R+1$ genes, for example, (0, $\left.i_{11}, i_{12}, i_{13}, 0, i_{21}, i_{22}, i_{23}, \ldots, 0, i_{R 1}, i_{R 2}, \ldots, 0\right)$. Different sequences represent different solutions. The initial 100 population is generated randomly, and individuals that could not meet the requirements of delivery time windows should be deleted and generated randomly again. Parent chromosomes are selected with roulette wheel selection according to the fitness function (13). The vehicle capacity constraint is integrated into the objective function. $M$, a pretty big number, represents the penalty coefficient when orders on a vehicle are more than $Q_{v}$ :

$$
\begin{aligned}
\min z= & \sum_{r=1}^{R} \sum_{i=0}^{m+1} \sum_{j=0}^{m+1} x_{i j r}\left(\frac{d_{i j}}{v}\right) \\
& +M \sum_{r=1}^{R} \max \left(\sum_{i=1}^{m} y_{i r} q_{i}-Q_{v}, 0\right) .
\end{aligned}
$$


Then partially matched crossover is adopted as crossover operator. The crossing points are selected by using the certain crossover probability and two children are generated. Afterwards, a nonzero gene is generated mutation randomly with the certain mutation probability, and the gene generated originally in the chromosomes changes to the one which mutates. Eligible children would substitute the parents whose fitness is very low, and a new generation is formed. Terminate the algorithm when it updates the set generations.

Since a delivery point has one reception box at least, the calculation of $t_{j}^{\text {road }}$ in RB is similar to that in AHD. However, it is a VRP problem because RB has no limitation on delivery time. The formulas are all the same except the constraint (11). It should be altered to $s_{i} \leq T$. The formulas will not be listed again. A simple genetic algorithm is employed to solve this VRP problem. The programming of genetic algorithm is similar to that of AHD except the elimination of the constraint-deleting the individuals that could not meet the requirements of delivery time windows.

The calculation of $t_{j}^{\text {road }}$ in CDPs is very different from that in AHD and RB. The reason is that a CDP could serve several delivery points at the same time as long as the distances between them are acceptable to customers. Besides, CDPs have no capacity constraints. Thus, it is reasonable to cluster the delivery points on the basis of Euclidean distance. In real life, the choice of CDPs involves a lot of commercial and environmental factors. To simplify the calculation process, the CDPs in the experiment are located in the centers of clusters. From this, $K$-means clustering algorithm is applied to this problem first.

Objective function:

$$
\sum_{i=1}^{n} \min _{j \in\{1,2, \ldots, k\}}\left\|o_{i}-P_{j}\right\|^{2}
$$

subject to

$$
\begin{gathered}
\bar{o}_{i}=\frac{1}{\left|L_{i}\right|} \sum_{o \in L_{i}} o \\
E=\sum_{i=1}^{k} \sum_{o \in L_{i}}\left|o-\bar{o}_{i}\right|^{2},
\end{gathered}
$$

where $o_{i}$ denotes the locations of delivery points, $P_{j}$ denotes the average value of cluster $L_{i}$, and $E$ is the sum of squared errors. Constraint (15) states the average value of updated clusters; constraint (16) states the calculation rule. The clustering centers are recognized as delivery points and the shortest path among them is solved with VRP model. In this way, a hierarchical procedure involving one heuristic and two algorithm phases is developed. Phase I aims to identify a set of feasible clusters with $k$-means algorithm while phase II assigns clusters to vehicles and sequences them on each tour by using VRP problem.

In short, conceptually, AHD's operation efficiency is lowest because of the long time waiting for customers while
CDPs' operation efficiency is the highest due to the simplest operational process. The average time per order is

$$
t_{j}=\frac{t_{j}^{\text {sum }}}{q} .
$$

Different modes lead to different operation efficiencies which result in different demands of equipment. Appropriate amount of equipment helps express companies accomplish tasks with minimal cost. The parameter $n_{j}$-number of equipment is the intermediate variable between operation efficiency and total cost. The relationship is

$$
\frac{q t_{j}}{n_{j}} \leq T
$$

which indicates all the arrival orders should be distributed within working time of the same day.

2.3. Cost Structure. Different modes have different cost components. There are some common cost components such as investments of vehicles, handheld terminals, and management expense. However even these common components have different values because the most appropriate device type and commission are diverse in different modes, only management expenses of three modes are the same. Notice that fuel fee is ignored in the model, because many express companies provide couriers with vehicles but are not responsible for their fuel fee in reality. In AHD, the total cost is

$$
C=M+n(c+w f)+w q .
$$

The total cost contains fixed administrative expense $M$, short fixed cost, and variable cost. Short fixed cost consists of the investment on devices $n * c$ and welfare payment for labors $n * w f$. An employee is assumed to operate a device. $c$ includes the price of a vehicle and a handheld terminal and their maintenance fee; $w$ indicates the commission per order paid to couriers.

In $\mathrm{RB}$, the total cost is

$$
C=M+n(c+w f)+w q+I .
$$

Total cost of RB should be added an item $I$ - the investment of purchasing and maintaining reception boxes on the basis of AHD.

In CDPs, the total cost is

$$
C=M+n(c+w f)+(w+\gamma) q+I .
$$

Total cost of CDPs has one more item that the commissions paid to the cooperators $\gamma * q$; $I$ is the expenditure of negotiating and signing contracts with cooperators.

From the formula above, only labors and cooperators commission are proportional to the quantity of orders. Administrative expense is a constant. Short fixed cost which includes investments on devices and welfare payments is a step function. This model reveals all detailed cost contained in the "last mile" delivery activities. 


\section{Numerical Experiments}

It is difficult to obtain the delivery time of the three modes in real life. On the one hand, it is hard to put the three modes into practice under the same circumstance to make the results comparable; on the other hand, it involves express companies' business secrets. In consequence, simulation experiments are made with the software Matlab. The certain crossover and mutation probability of genetic algorithm are 0.8 and 0.2 , respectively, and the algorithm is terminated when it updates 200 generations.

In the specific experimental, 30 delivery points represent 30 communities. The locations of delivery points are generated randomly in the square of 25 kilometers, and it is suitable for China's condition. Demand of every delivery point is generated randomly too. Other data of constants were collected from the investigations on couriers and interviews with middle-level managers from ShenTong Express, YuanTong Express, ZTO Express, YunDa Express, and SF Express which are the main e-commerce logistics providers in China.

To simplify the simulation process, the depot is set up in the centre of this area. Every vehicle starts off and comes back here. The roads between any two points are assumed to be straight-lines. On the first scenario, demands of all communities are set less than 36 orders to make sure setting up a reception box in a community is enough, and this scenario is regarded as the standard scenario.

3.1. The Importance of Vehicle Choice. To make the results of three modes comparable and show the significance of employing the most appropriate vehicles, we compare the effects of different vehicles applied to AHD. In china, seldom express companies offer delivery time windows to choose and just few customers ask for delivery time windows according to the investigation mentioned above. Here are the limiting values of the vehicle fleet. The first are the limiting values of electrotricycles in AHD:

(1) maximum 300 orders per electrotricycle,

(2) average velocity: $30 \mathrm{~km} / \mathrm{h}$,

(3) working time maximum 10 hours per electrotricycle,

(4) stopping and starting the electrotricycle: 2 min every time,

(5) serving per customer: $5 \mathrm{~min}$,

(6) 3 delivery points ask for an hour time window (11:3012:30, 14:00-15:00, and 17:30-18:30, resp.).

Second are the limiting values of light-weight vans which are used in RB and CDPs:

(1) maximum 800 orders per van,

(2) average velocity: $60 \mathrm{~km} / \mathrm{h}$,

(3) working time maximum 10 hours per van,

(4) stopping and starting the van: 2 min every time,

(5) serving per customer: $5 \mathrm{~min}$,

(6) 3 delivery points ask for an hour time window (11:3012:30, 14:00-15:00, and 17:30-18:30, resp.).
TABLE 1: Time spent situation in AHD with two kinds of vehicles.

\begin{tabular}{lcc}
\hline \multirow{2}{*}{ Items } & \multicolumn{2}{c}{ Modes } \\
& Electrotricycle & Light-weight van \\
\hline Total distance $(\mathrm{km})$ & 42.16 & 41.65 \\
Number of orders & 744 & 744 \\
Stopping times & 30 & 30 \\
Number of vehicles & 8 & 8 \\
$t^{\text {road }}($ min) & 84.3 & 41.7 \\
$t^{\text {s }}$ (min) & 60 & 60 \\
$t^{\text {service }}($ min) & 3720 & 3720 \\
Buffering time (min) & 20 & 20 \\
Total time (min) & 3884 & 3841.7 \\
Average time per order (min) & 5.22 & 5.16 \\
\hline
\end{tabular}

The delivery situations with the two kinds of vehicles in AHD are displayed in Figure 1.

Parameter values are displayed in Table 1 . The efficiency of employing light-weight vans is just a little bit higher than that of electrotricycles, because the former one has larger average velocity and spends less time on the road. $t^{s}$ and $t^{\text {service }}$ are exactly the same. Time spent on serving customers occupies the largest proportion of total time. Thus no matter how fast and how big the vehicle is, delivery efficiency could not be improved visibly. That is why the routes in two cases are similar.

Daily total cost of the two cases is calculated on the basis of the delivery efficiency and cost structure. All the conditions are set exactly the same as Section 3.3.

The cost of using electrotricycles and light-weight vans in AHD is showed in Figure 2. It is found that the cost of employing light-weight vans is always higher, because the delivery efficiency of employing light-weight vans could not be improved largely while the investment is much more. As the increase of orders, the gap between them is bigger and bigger. That is mainly because the growth of orders makes $t^{\text {service }}$ occupy a larger proportion of total time and leads to more and more advantages of employing light-weight vans disappeared, but the investment of light-weight vans is more and more greater than that of electrotricycles.

From the foregoing, the use of appropriate vehicles in different delivery modes is very important. In AHD, electrotricycle is suitable, but in RB and CDPs, light-weight van is preferable.

3.2. Delivery Efficiencies of Three Modes. According to the analysis of Section 3.1, light-weight vans are employed in RB. The limiting values of the vehicle fleet in $\mathrm{RB}$ are listed as follows:

(1) maximum 800 orders per van,

(2) average velocity: $60 \mathrm{~km} / \mathrm{h}$,

(3) working time maximum 10 hours per van,

(4) stopping and starting the van: 2 min every time,

(5) serving per customer: $1 \mathrm{~min}$. 


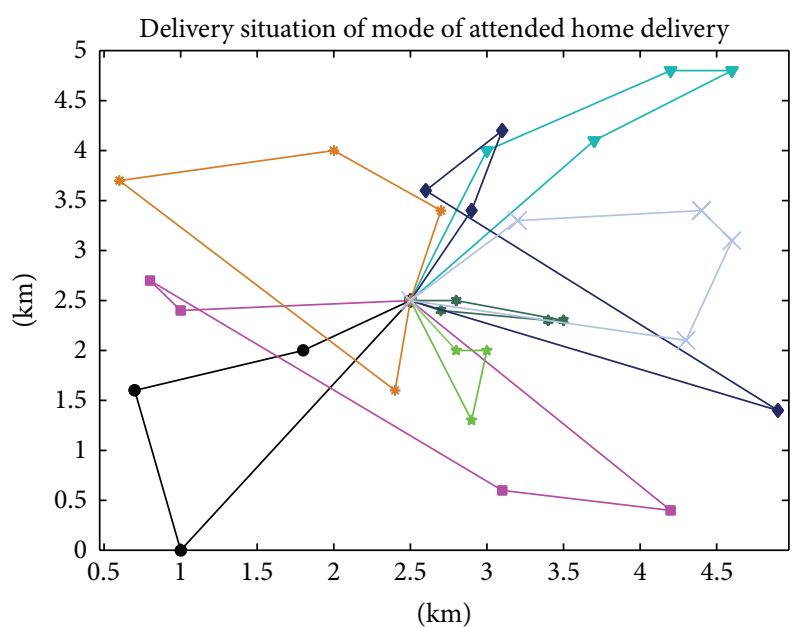

(a) With electrotricycles

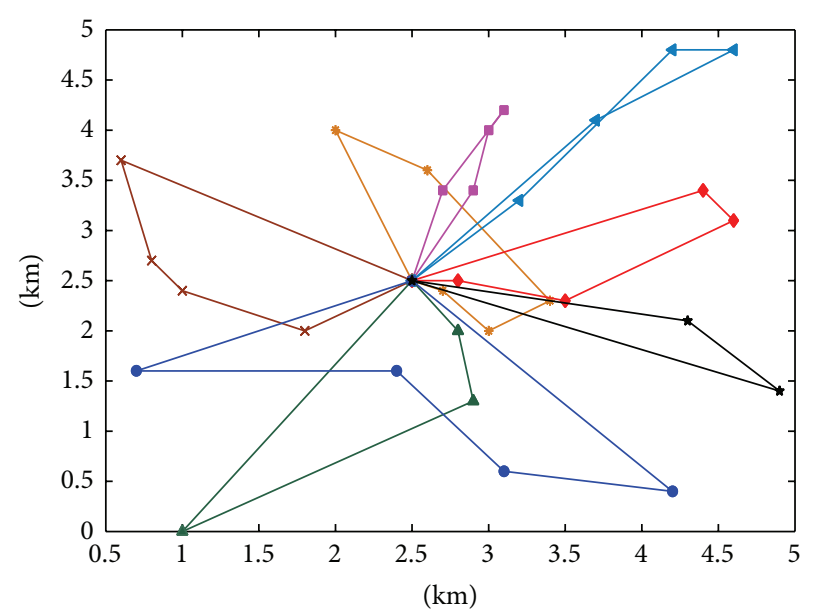

(b) With light-weight vans

Figure 1: Delivery situation of AHD with two kinds of vehicles.

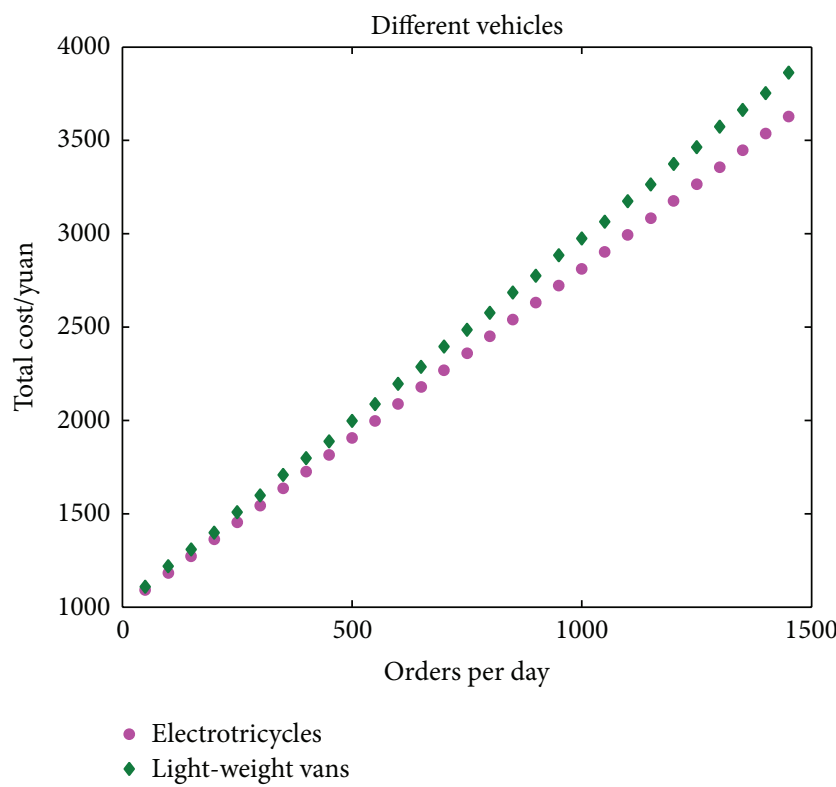

FIgURE 2: Total cost of AHD with two kinds of vehicles.

It could be seen AHD needs much more vehicles and couriers than RB by comparing Figure 1 with Figure 3.

As mentioned above, 30 delivery points should be clustered first in CDPs. Since the scope is a square of 25 kilometers, it's enough to set up 5 collection-and-delivery points. The result is displayed in Figure 4. The points of the same shape are in the same cluster. The big green dot represents DC while the black crosses stand for CDPs. It could be seen that the distance between every delivery point and its nearest CDP is no more than $1 \mathrm{~km}$ (a reasonable distance that conforms to the key successful rule [15]). The limiting values of the vehicle fleet in CDPs are listed as follows:

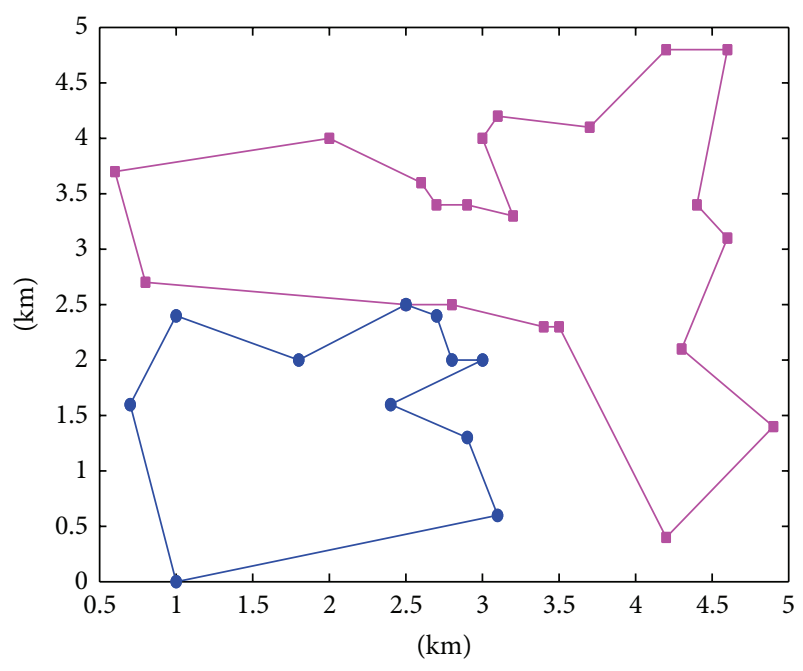

FIGURE 3: Delivery situation of RB.

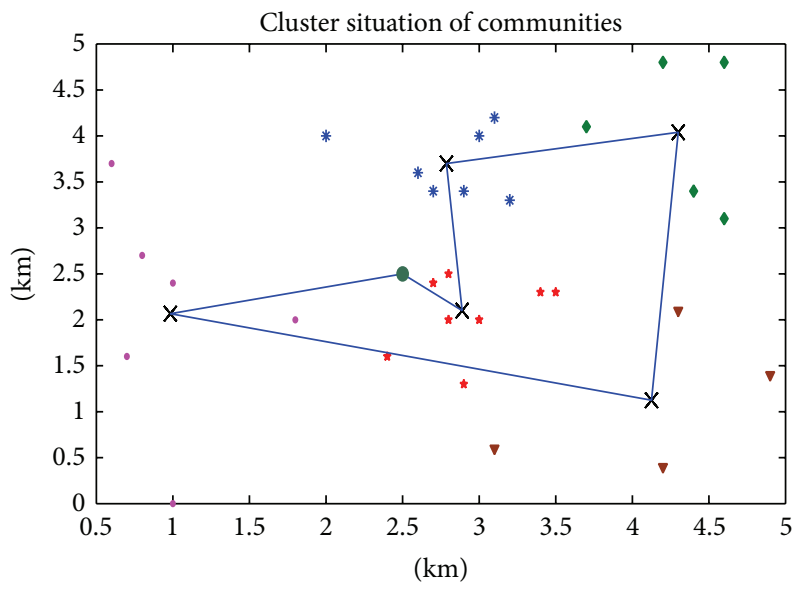

Figure 4: Cluster situation of communities. 


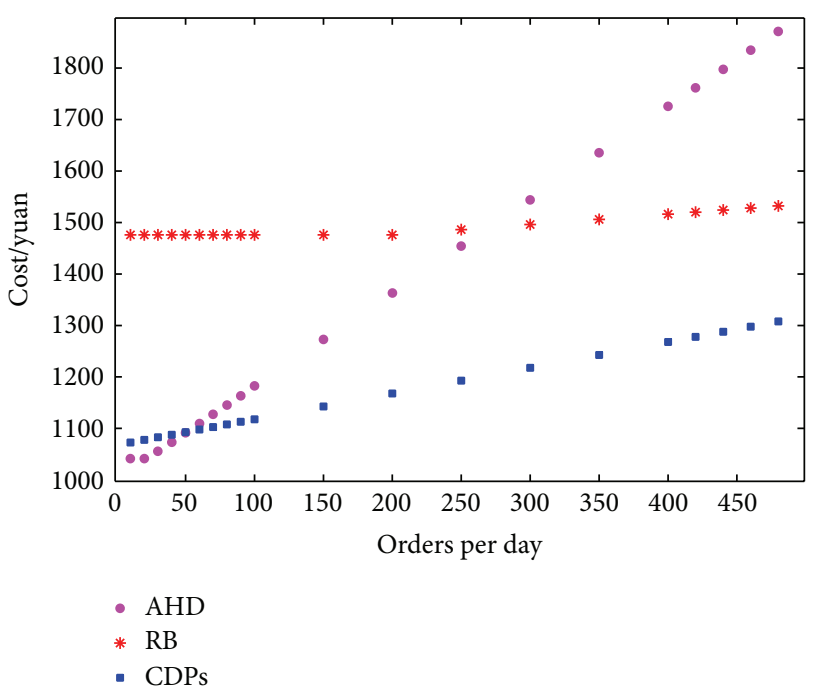

Figure 5: Total cost of three modes.

TABLE 2: Time spent situation in standard scenario.

\begin{tabular}{lccc}
\hline Items & \multicolumn{3}{c}{ Modes } \\
& AHD & RB & CDPs \\
\hline Total distance $(\mathrm{km})$ & 42.16 & 26.9 & 11.5 \\
Number of orders & 744 & 744 & 744 \\
Stopping times & 30 & 30 & 5 \\
Number of vehicles & 8 & 2 & 1 \\
$t^{\text {road }(\text { min) }}$ & 84.3 & 26.9 & 11.5 \\
$t^{s}$ (min) & 60 & 60 & 20 \\
$t^{\text {service }(\min )}$ & 3720 & 744 & 148.8 \\
Buffering time (min) & 20 & 20 & 20 \\
Total time (min) & 3884 & 850 & 200.3 \\
Average time per order (min) & 5.22 & 1.15 & 0.27 \\
\hline
\end{tabular}

(1) maximum 800 orders per van,

(2) average velocity: $60 \mathrm{~km} / \mathrm{h}$,

(3) working time maximum 10 hours per van,

(4) stopping and starting the van: 4 min every time,

(5) serving per customer: $0.2 \mathrm{~min}$.

The result is displayed in Figure 4 as well. As expected, total travel distance in CDPs decreases sharply compared to that in AHD and RB.

To all the three modes, $20 \mathrm{~min}$ is endowed as buffering time to make up for the time couriers spending doing other things. The values of parameters in the three modes are showed in Table 2.

From Table 2, CDPs have the highest operation efficiency, followed by RB and then AHD. $t^{\text {service }}$ occupies the largest proportion of total time. That is mainly because every community's demand is relatively large and residential density in this region is high.

The distinct of operation efficiency reflected is evident. Obviously, CDPs need the least $t^{\text {service }}$ while AHD needs the most. Because of AHD, couriers have to wait for the receiver
TABLE 3: Time spent situation with half orders compared with standard scenario.

\begin{tabular}{lccc}
\hline Items & \multicolumn{3}{c}{ Modes } \\
& AHD & RB & CDPs \\
\hline Total distance $(\mathrm{km})$ & 42.16 & 26.9 & 11.5 \\
Number of orders & 372 & 372 & 372 \\
Stopping times & 30 & 30 & 5 \\
Number of vehicles & 8 & 2 & 1 \\
$t^{\text {road }}(\min )$ & 84.3 & 26.9 & 11.5 \\
$t^{s}($ min) & 60 & 60 & 20 \\
$t^{\text {service }}(\min )$ & 1860 & 372 & 74.4 \\
Buffering time $(\min )$ & 20 & 20 & 20 \\
Total time (min) & 2024.3 & 478.9 & 125.9 \\
Average time per order $(\min )$ & 5.44 & 1.29 & 0.34 \\
\hline
\end{tabular}

TABLE 4: Time spent situation with double orders compared with standard scenario.

\begin{tabular}{lccc}
\hline Items & \multicolumn{3}{c}{ Modes } \\
& AHD & RB & CDPs \\
\hline Total distance $(\mathrm{km})$ & 60.1 & 29.9 & 11.5 \\
Number of orders & 1488 & 1488 & 1488 \\
Stopping times & 30 & 30 & 5 \\
Number of vehicles & 14 & 3 & 1 \\
$t^{\text {road }}($ min) & 120.2 & 29.9 & 11.5 \\
$t^{s}$ (min) & 60 & 60 & 20 \\
$t^{\text {service }}($ min) & 7440 & 1488 & 297.6 \\
Buffering time (min) & 20 & 20 & 20 \\
Total time (min) & 7640.2 & 1597.9 & 349.1 \\
Average time per order (min) & 5.13 & 1.07 & 0.23 \\
\hline
\end{tabular}

to sigh for the goods and sometimes wait for longer till the receiver checks the goods. In RB, couriers put the goods in the corresponding boxes and scan them. Apparently, this would be faster. Furthermore, couriers just unload and scan goods. Besides, both CDPs and RB modes save couriers' walking time because unified delivery points always locate on the first floor and enjoy convenient transportation.

CDPs would cost the least $t^{s}$ due to its much less parking times. RB needs more $t^{s}$ than CDPs but less than AHD. $t^{s}$ is affected by residential density [4] and the ease of parking. For the growth of private cars in cities, perhaps the problem of parking would be another big obstacle to elogistics increasingly. From the perspective of this point, RB and CDPs would be more advantageous in future.

AHD spends much more $t^{\text {road }}$ than RB and CDPs. That happens mainly because the vehicles suitable for AHD go slower and vehicles have to go through extra way to satisfy customers' delivery time windows [29]. Consequently, $t^{\text {road }}$ is closely connected with residential density and customers' delivery time windows in AHD [5].

To study the influence of orders on operation efficiency, the parameters are calculated in half and double orders, respectively. The results are shown in Tables 3 and 4. 


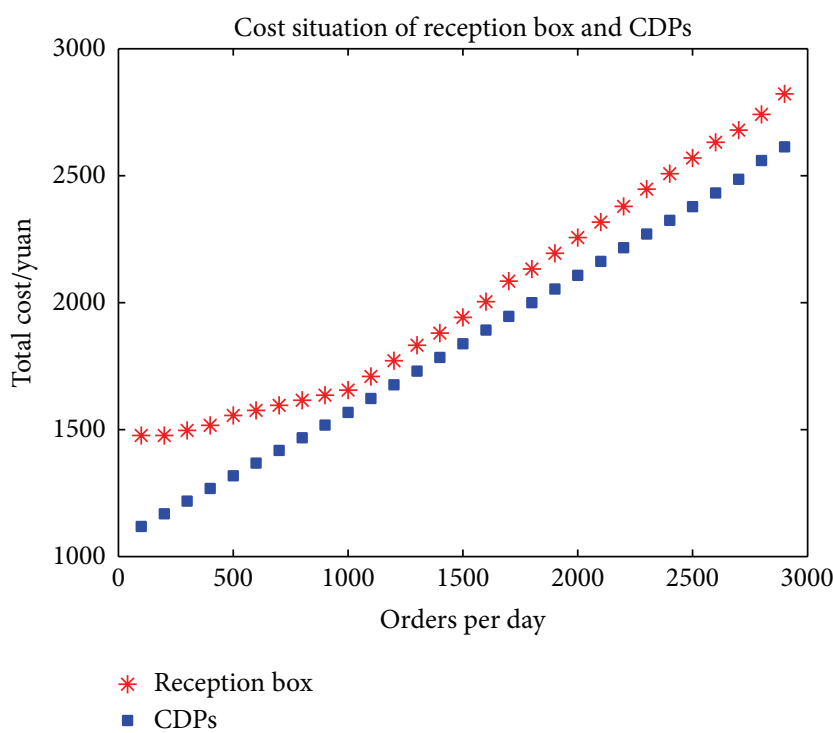

(a) $\gamma=0.5, I_{\text {each }}=5000$

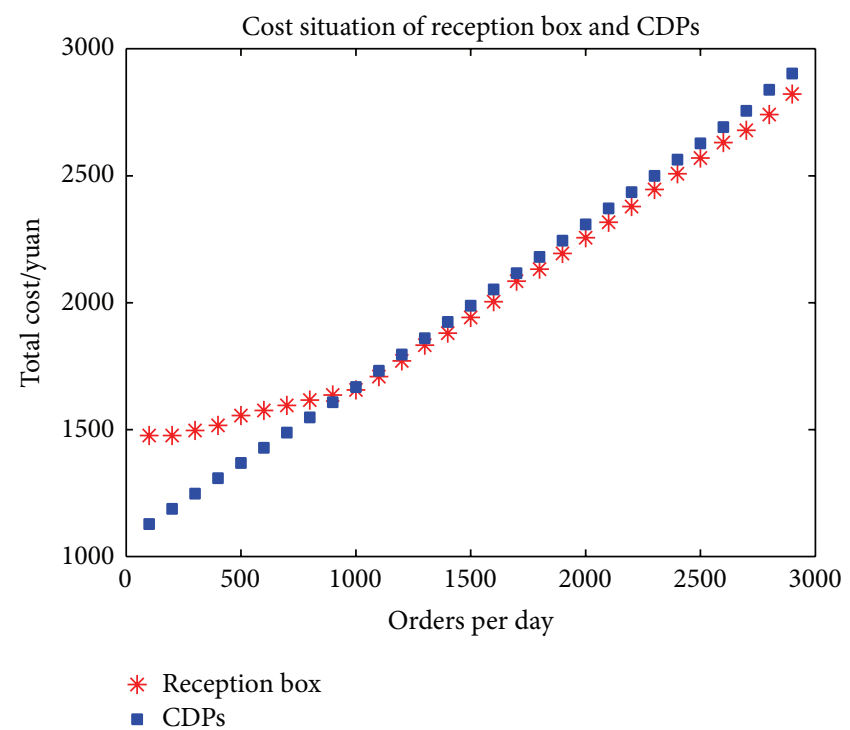

(b) $\gamma=0.6, I_{\text {each }}=5000$

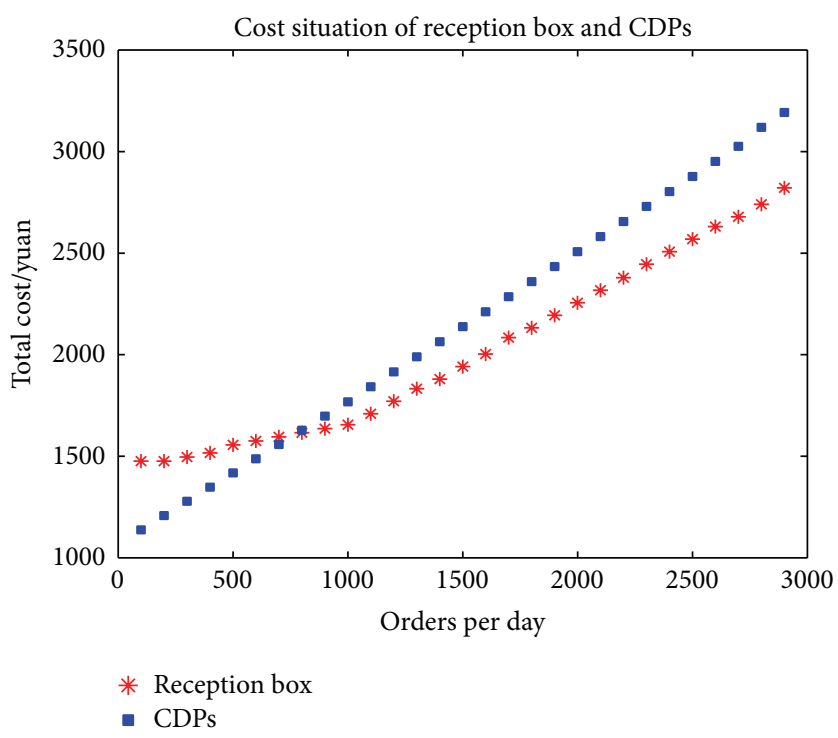

(c) $\gamma=0.7, I_{\text {each }}=5000$

FIgURE 6: Cost situation of RB and CDPs with changing third party commission.

Visually, the average time per order of each mode changes synchronously with orders. That is because $t^{\text {road }}$ and $t^{s}$ could be shared with more orders when orders increase. When only order quantity changes in the area, $t^{\text {road }}$ would change but not too much, resulting from the additional vehicles' extra way connected to the depot; $t^{s}$ would be relatively fixed; $t^{\text {service }}$ would increase or decrease in proportion with order quantity. In three scenarios, the ratios of average time per order in three modes are $1: 0.24: 0.063,1: 0.22: 0.052$, and $1: 0.21: 0.045$, corresponding to 372,744 , and 1488 orders, respectively. The result indicates that CDPs' operation efficiency will be more and more competitive as the increase of orders. It mainly benefits from the high efficiency of serving customers and no limit to the CDPs' capacity.
3.3. Total Cost of Three Modes. The total cost of every mode is showed in Figure 5. It clearly reflects higher operation efficiency does not mean lower cost through fair and foul. From Figure 5, it could be concluded when daily demand is less than 50 orders in this region, adopting AHD is wise. At this time, CDPs cost a little higher than AHD while RB costs a great deal. That is because the initial fixed investment of reception boxes is very large, and the vehicles used in $\mathrm{RB}$ and CDPs cost a lot more than that in AHD. On account of AHD's high variable cost and low operation efficiency, its cost soars sharply as the increase of orders. On the contrary, the variable cost of RB and CDPs is much smaller.

When orders are massive, AHD definitely costs the most, but which one costs the least still needs to be studied 


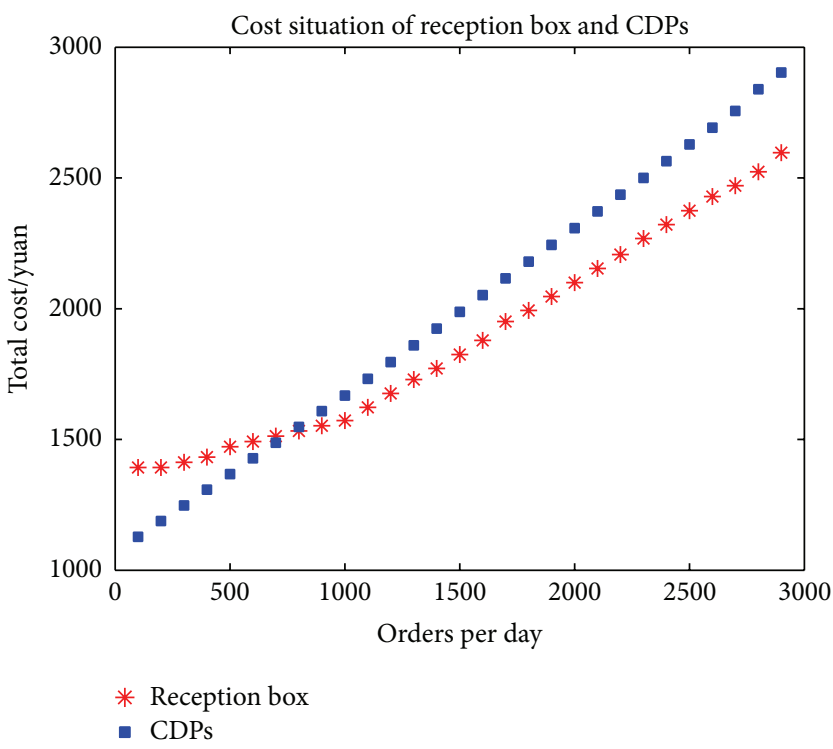

(a) $\gamma=0.6, I_{\text {each }}=4000$

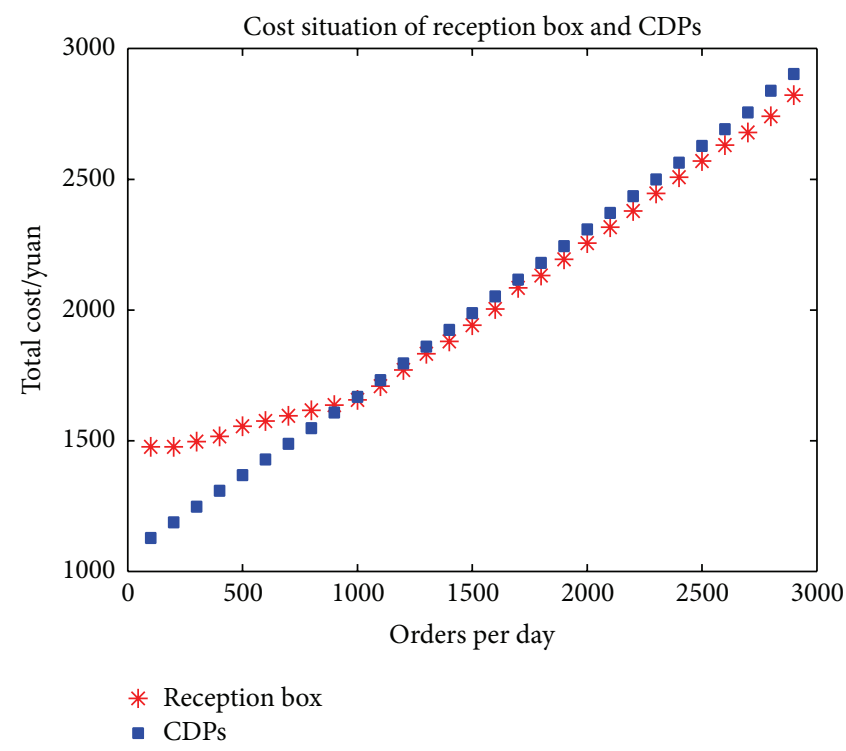

(b) $\gamma=0.6, I_{\text {each }}=5000$

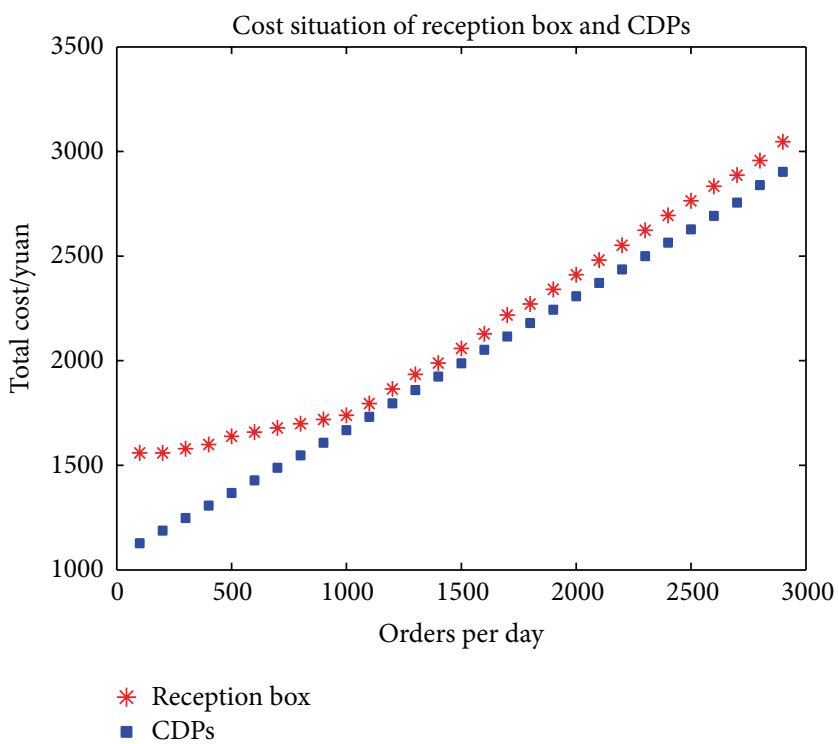

(c) $\gamma=0.6, I_{\text {each }}=6000$

FIgURE 7: Cost situation of RB and CDPs with changing reception box expense.

further. According to Figures 6 and 7, the choice of RB or CDPs depends on the expense of reception boxes and the commission paid to the third party partners, because when orders increase, the extra investment of new reception boxes is the main incremental cost in $\mathrm{RB}$ while the commission paid to third party partners is the main incremental cost in CDPs. Figure 6 shows the cost situation with stable expense of reception box and changing third party commission while Figure 7 shows the cost situation on the contrary. $I_{\text {each }}$ denotes the expense of a reception box per year. It could be concluded that the higher the commission paid to third party partners is, the faster the cost of CDPs would exceed that of RB. When third party commission is small, cost of CDPs will be always less than that of RB. However, even when such thing happens, it does not mean RB is utterly useless, because it has some unique advantages, such as providing refrigeration service and being set up in luxury residential where couriers are not allowed to enter.

In general, when orders are not many, it is wise to adopt AHD; when orders are massive enough, it is wise to adopt RB and CDPs, but which one to choose depends on the current context. In addition to this, cooperating with e-logistics providers benefits third party partners, as researchers point out that one in four customers would make a purchase in the store when collecting or returning their parcels [13]. It means CDPs are expected to become the most economical way when 
orders are massive through cutting down commissions paid to third party partners counting on the benefits they have already made.

\section{Conclusions}

Despite the three "last mile" delivery modes are concurrence in practice, a little theoretical research aims at choosing them according to different scenarios so far. This paper makes the quantitative study of different delivery modes' competitiveness through analyzing "last mile" activities' cost structure and operation efficiency in different scenarios.

The major conclusions of this paper are concluded as follows. (i) Total cost of "last mile" delivery relies on the price of manpower and material resources and operation efficiency. Additionally, different "last mile" delivery modes have different operation efficiencies. Furthermore, choosing the most appropriate devices reduces cost effectively. (ii) Operation efficiency is affected by order quantity, and CDPs are more sensitive to the change of orders. Also, under normal circumstances, CDPs' operation efficiency is the highest, followed by RB and then AHD. (iii) When orders are few, AHD is the most suitable mode as the result of the larger amount of equipment investment in RB and CDPs. On the contrary, when there are plenty of orders in high residential density areas with high reception box expense and low commission of third party partners, CDPs are optimum; moreover when there are plenty of orders in sparse areas or high-grade district, RB has the optimal advantage.

In summary, the paper extends the academic study of "last mile" delivery, puts forward the cost structure of each mode, then reveals the variation tendency of cost along with the change of order quantity, and further analyzes the competitiveness of each mode in different scenarios. It could provide some theoretical guidance for e-logistic providers.

Given the fact that the use of RB and CDPs is still both at the very beginning, with excellent prospect for increasing use of these concepts in the future, more quantitative researches on this subject are necessary. Customers' satisfaction varies with each individual and this should be taken into consideration in the near future research on this field. Besides that those modes are considered separately in this paper, in reality, they could be applied mixed in the same district and that should be figured out by researchers.

\section{Conflict of Interests}

The authors declare that there is no conflict of interests regarding the publication of this paper.

\section{Acknowledgments}

The authors would like to thank the anonymous referees and Professor Kwok-Wo Wong for their useful comments and suggestions, which were very helpful in improving this paper. This work was supported by the National Natural Science Foundation of China under Grant (nos. 71171029, 71271037, 71201014, and 71301020) and the China Scholarship Council.

\section{References}

[1] J.-C. Pai and C.-H. Yeh, "Factors affecting the implementation of e-business strategies: an empirical study in Taiwan," Management Decision, vol. 46, no. 5, pp. 681-690, 2008.

[2] China Internet Network Information Center, "China's online shopping market research report in 2012," April 2007 (Chinese), http://www.cnnic.cn/hlwfzyj/hlwxzbg/dzswbg/201304/t20130417_ 39290.htm.

[3] China Enterprise News, "Optimization of logistics distribution chain, solve last mile delivery problem," September 2013 (Chinese), http://info.jctrans.com/news/synthetic_trans/ 20139121967744.shtml.

[4] V. Kupke, P. Rossini, and S. McGreal, "Measuring the impact of higher density housing development," Property Management, vol. 30, no. 3, pp. 274-291, 2012.

[5] P. J. Smailes, N. Argent, and T. L. C. Griffin, "Rural population density: its impact on social and demographic aspects of rural communities," Journal of Rural Studies, vol. 18, no. 4, pp. 385404, 2002.

[6] K. K. Boyer, A. M. Prud'homme, and W. Chung, "The last mile challenge: evaluating the effects of customer density and delivery window patterns," Journal of Business Logistics, vol. 30, no. 1, pp. 185-201, 2009.

[7] N. Agatz, A. Campbell, M. Fleischmann, and M. Savelsbergh, "Time slot management in attended home delivery," Transportation Science, vol. 45, no. 3, pp. 435-449, 2011.

[8] A. V. Hill, J. M. Hays, and E. Naveh, "A model for optimal delivery time guarantees," Journal of Service Research, vol. 2, no. 3, pp. 254-264, 2000.

[9] M. A. Bushuev and A. L. Guiffrida, "Optimal position of supply chain delivery window: concepts and general conditions," International Journal of Production Economics, vol. 137, no. 2, pp. 226-234, 2012.

[10] A. M. Campbell and M. Savelsbergh, "Incentive schemes for attended home delivery services," Transportation Science, vol. 40, no. 3, pp. 327-341, 2006.

[11] M. Punakivi, H. Yrjölä, and J. Holmström, "Solving the last mile issue: reception box or delivery box," International Journal of Physical Distribution \& Logistics Management, vol. 31, no. 6, pp. 427-439, 2001.

[12] M. Punakivi and K. Tanskanen, "Increasing the cost efficiency of e-fulfilment using shared reception boxes," International Journal of Retail \& Distribution Management, vol. 30, no. 10, pp. 498-507, 2002.

[13] J. W. J. Weltevreden, "B2c e-commerce logistics: the rise of collection-and-delivery points in The Netherlands," International Journal of Retail \& Distribution Management, vol. 36, no. 8, pp. 638-660, 2008.

[14] H. L. Lee and S. Whang, "Winning the last mile of e-commerce," MIT Sloan Management Review, vol. 42, no. 4, pp. 54-62, 2001.

[15] L. Song, T. Cherrett, F. McLeod, and W. Guan, "Addressing the last mile problem-the transport impacts of collection/delivery points," in Proceedings of the 88th Annual Meeting of the Transportation Research Board, 2009.

[16] F. McLeod, T. Cherrett, and L. Song, “Transport impacts of local collection/delivery points," International Journal of Logistics, vol. 9, no. 3, pp. 307-317, 2006.

[17] Y. Yi and T. Gong, "The effects of customer justice perception and affect on customer citizenship behavior and customer dysfunctional behavior," Industrial Marketing Management, vol. 37, no. 7, pp. 767-783, 2008. 
[18] Z. Ursani, D. Essam, D. Cornforth, and R. Stocker, "Localized genetic algorithm for vehicle routing problem with time windows," Applied Soft Computing, vol. 11, no. 8, pp. 5375-5390, 2011.

[19] R. Liu, X. Xie, V. Augusto, and C. Rodriguez, "Heuristic algorithms for a vehicle routing problem with simultaneous delivery and pickup and time windows in home health care," European Journal of Operational Research, vol. 230, no. 3, pp. 475-486, 2013.

[20] V. Pureza, R. Morabito, and M. Reimann, "Vehicle routing with multiple deliverymen: modeling and heuristic approaches for the VRPTW," European Journal of Operational Research, vol. 218, no. 3, pp. 636-647, 2012.

[21] J. Ruan, X. Wang, Y. Shi, and Z. Sun, "Scenario-based path selection in uncertain emergency transportation networks," International Journal of Innovative Computing, Information \& Control, vol. 9, no. 8, pp. 3293-3305, 2013.

[22] X. Wang, J. Ruan, and Y. Shi, "A recovery model for combinational disruptions in logistics delivery: considering the real-world participators," International Journal of Production Economics, vol. 140, no. 1, pp. 508-520, 2012.

[23] F. P. Goksal, I. Karaoglan, and F. Altiparmak, "A hybrid discrete particle swarm optimization for vehicle routing problem with simultaneous pickup and delivery," Computers \& Industrial Engineering, vol. 65, no. 1, pp. 39-53, 2013.

[24] J. Ruan, X. Wang, and Y. Shi, "Developing fast predictors for large-scale time series using fuzzy granular support vector machines," Applied Soft Computing, vol. 13, no. 9, pp. 3981-4000, 2012.

[25] R. Dondo and J. Cerdá, "A cluster-based optimization approach for the multi-depot heterogeneous fleet vehicle routing problem with time windows," European Journal of Operational Research, vol. 176, no. 3, pp. 1478-1507, 2007.

[26] G. di Fatta, F. Blasa, S. Cafiero, and G. Fortino, "Fault tolerant decentralised K-means clustering for asynchronous large-scale networks," Journal of Parallel and Distributed Computing, vol. 73, no. 3, pp. 317-329, 2013.

[27] F. An and H. J. Mattausch, "K-means clustering algorithm for multimedia applications with flexible HW/SW co-design," Journal of Systems Architecture, vol. 59, no. 3, pp. 155-164, 2012.

[28] J. Ruan, X. Wang, and Y. Shi, "A clustering-based approach for emergency medical supplies transportation in large-scale disasters," ICIC Express Letters, vol. 8, no. 1, pp. 187-193, 2014.

[29] M. Punakivi and J. Saranen, "Identifying the success factors in e-grocery home delivery," International Journal of Retail \& Distribution Management, vol. 29, no. 4, pp. 156-163, 2001. 


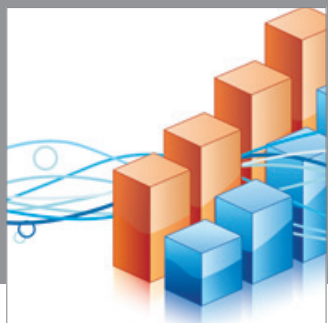

Advances in

Operations Research

mansans

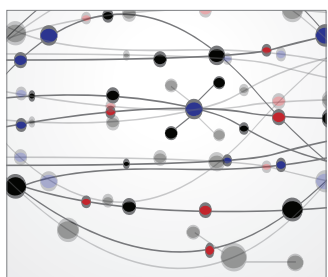

The Scientific World Journal
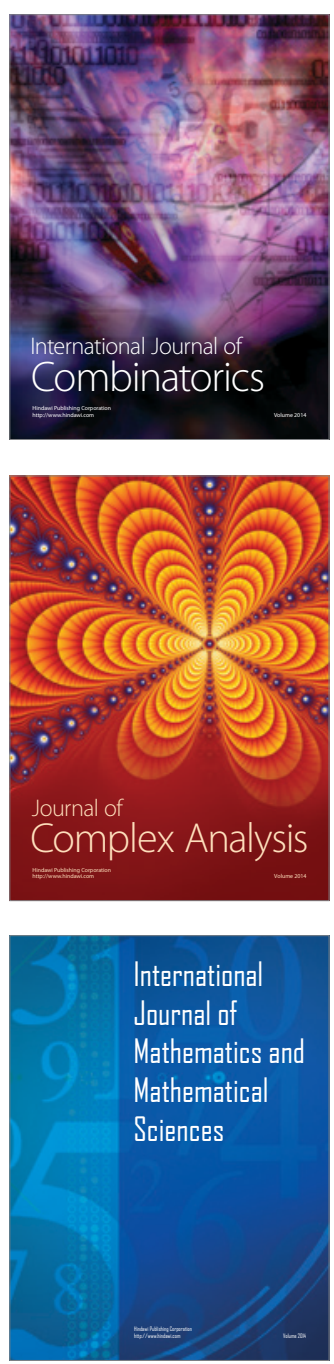
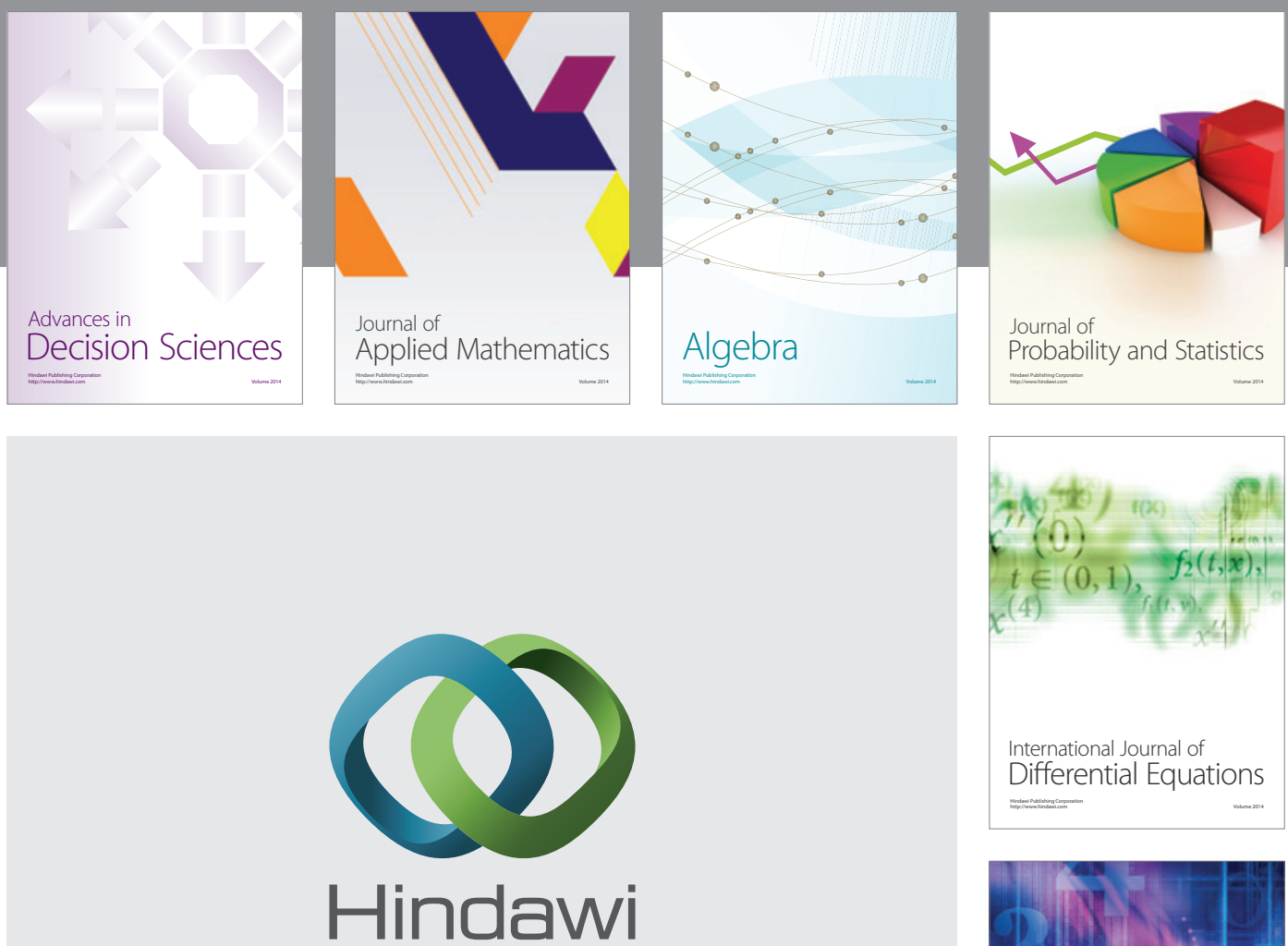

Submit your manuscripts at http://www.hindawi.com
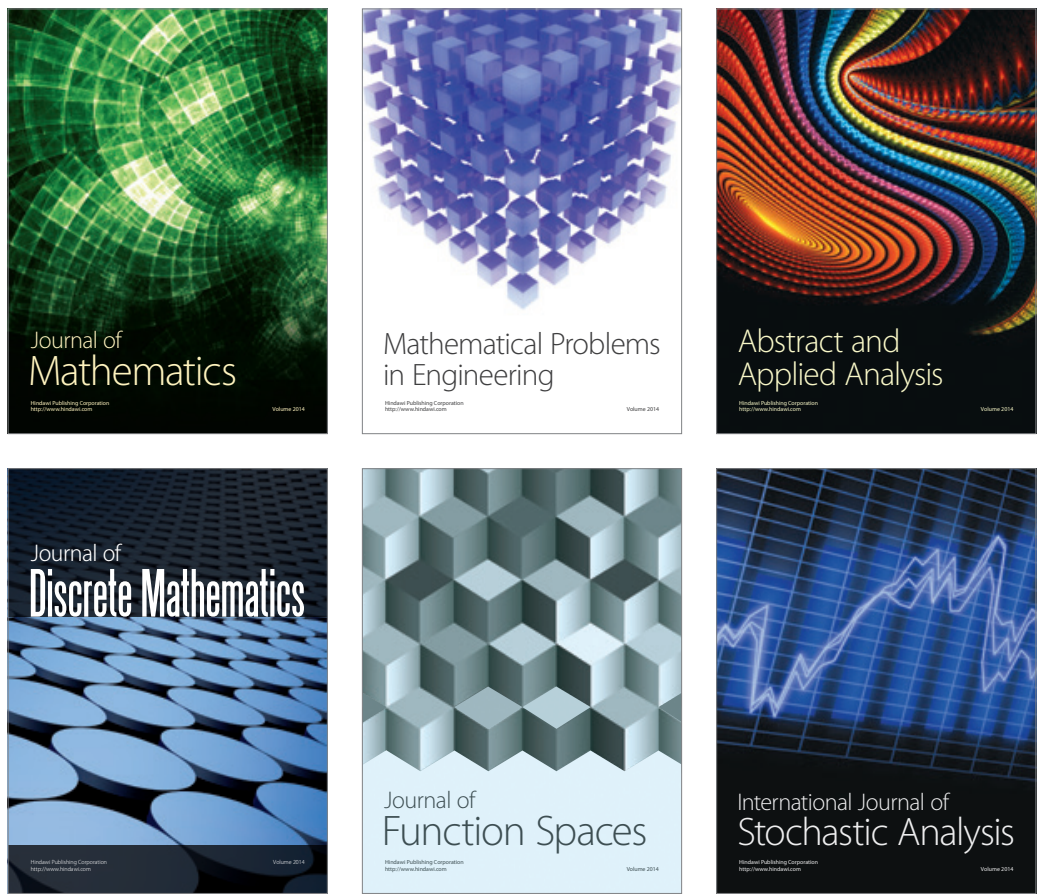

Journal of

Function Spaces

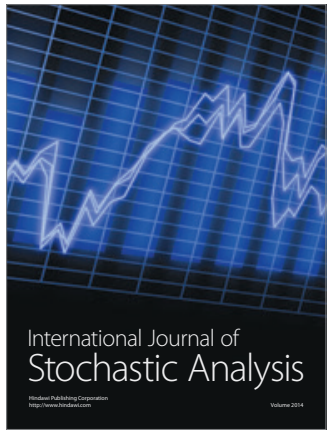

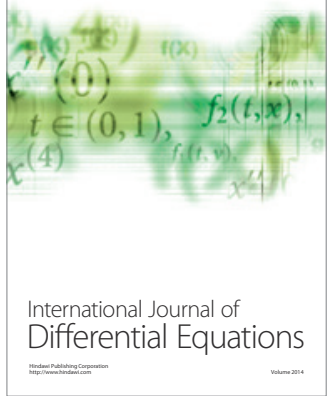
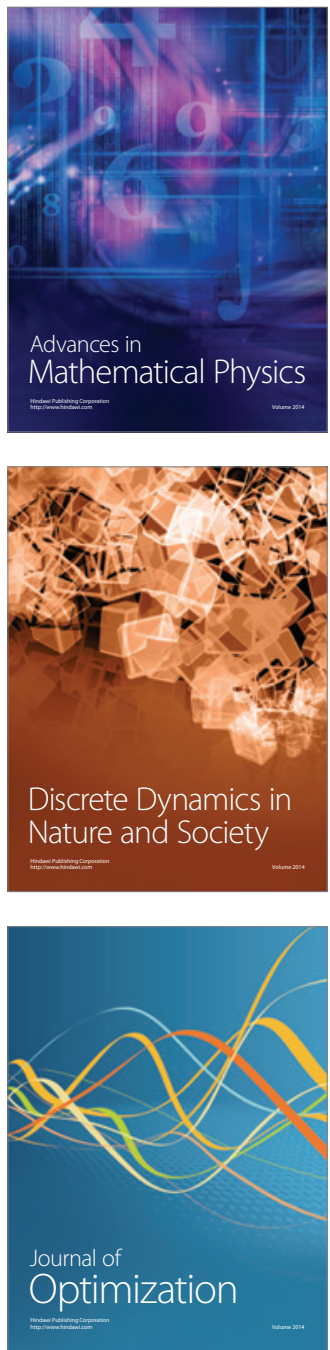